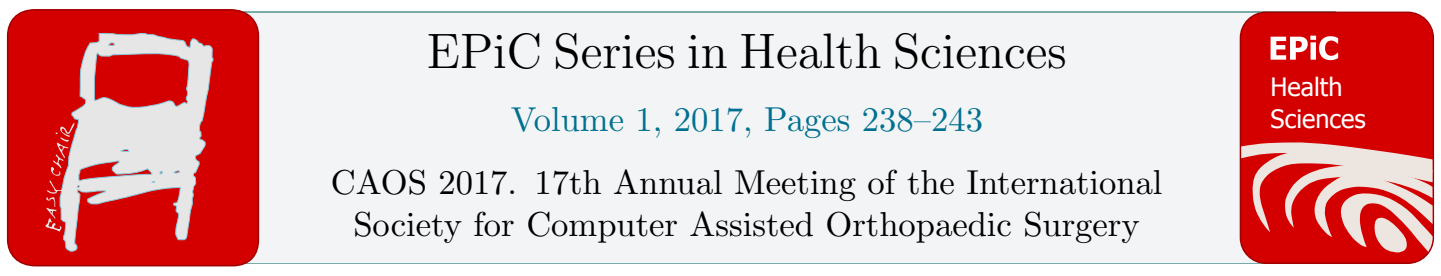

\title{
Analysis of Gender-Specific Aspects of the Morphology of the Distal Femur
}

\author{
Malte Asseln $^{1 *}$, Christoph Hänisch ${ }^{1}$, Fabian Schick ${ }^{1}$ and Klaus \\ Radermacher $^{1}$ \\ ${ }^{1 *}$ Chair of Medical Engineering, Helmholtz Institute for Biomedical Engineering, RWTH Aachen \\ University, Aachen, 52074, Germany \\ asseln@hia.rwth-aachen.de
}

\begin{abstract}
In total knee arthroplasty (TKA) the implant design is one key factor for a proper functional restoration of the diseased knee. Therefore, detailed knowledge on the shape (morphology) is essential to guide the design process. In literature, the morphology has been extensively studied revealing differences, e.g. between ethnicity and gender. However, it is still unclear in which way gender-specific morphological differences are sexual dimorphism or explained by differences in size.

The aim of this study was to investigate the morphology of the distal femur under gender-specific aspects for a large group of patients. Statistical analysis was used to reveal significant differences and subsequent correlation analysis to normalize the morphology.

A dataset of $n=363$ segmented distal femoral bone surface reconstructions (229 female, 134 male) were randomly collected from a database of patients which underwent TKA. In total, 34 morphological features (distances, angles), quantifying the distal femoral geometry, were determined full automatically. Subsequently, graphs and descriptive statistics were used to check normality and gender-specific differences were analyzed by calculating the $95 \%$ confidence intervals for women and men separately. Finally, significant differences were normalized by dividing each feature by appropriate distance measurements and confidence intervals were recalculated.

Looking at the confidence $95 \%$ intervals, 6 of 34 features did not show any significant differences between genders. Remarkably, this primarily involves angular (relative) features whereas distance (absolute) measurements were mostly gender dependent. Then, we normalized all distance measurements and radii according to their direction of measurement: Features defined in medial/lateral (ML) direction were divided by the overall ML width and those following the anterior/posterior direction were normalized based on the overall AP length. The results demonstrated that genderspecific differences mostly disappear by using an adequate normalization term.
\end{abstract}


In conclusion, implant sizes (femoral components) should not be linearly scaled according to one dimension. Instead, ML and AP directions should be regarded separately (non-isotropic scaling). Taking this into consideration, gender-specific differences might be neglected.

\section{Introduction}

In total knee arthroplasty (TKA) the implant design is one key factor for a proper functional restoration of the diseased knee. Therefore, detailed knowledge on the shape (morphology) is essential to guide the design process. In literature, the morphology has been extensively studied revealing differences, e.g. between ethnicity and gender (Guy et al., 2012; Yang et al., 2014). However, it is still unclear in which way gender-specific morphological differences are sexual dimorphism or differences in size and potentially can be eliminated by one or several scaling factors.

Already in 1913 Parsons et al. investigated the characteristics of the femur and reported gender differences in overall size (Parsons, 1914). Hitt et al. analysed anthropometric measurements collected from 337 knees after bone resection for TKA and found that some implant dimensions differed from the actual morphology (Hitt et al., 2003). These findings motivated the development of gender-specific implants. The design modifications concern the medial/lateral (ML) and anterior/posterior (AP) dimensions and the patellar shield of the femoral component. However, Mensch and Amstutz used 16 linear dimensions in 30 cadaveric knees based on 2D radiographs to analyse interrelationships. They concluded, that the ML width of the femur showed the highest correlations with other dimensions (Mensch and Amstutz, 1975).

The aim of this study was to investigate the morphology of the distal femur under gender-specific aspects for a large group of patients. Statistical analysis was used to reveal significant differences and subsequent correlation analysis to normalize the morphology.

\section{Materials and Methods}

A dataset of $n=363$ segmented distal femoral bone surface reconstructions (229 female, 134 male) were randomly collected from a database of patients which underwent TKA. Osteophytes were semiautomatically removed by clinical experts to ensure representative surface geometries. A selfdeveloped computational framework in MATLAB has been used, previously reported by our group (Asseln et al., 2016). In total, 34 morphological features (distances, angles), quantifying the distal femoral geometry, were determined full automatically (Figure 1). Subsequently, graphs and descriptive statistics were used to check normality, being a prerequisite for using mean and standard deviation for representative data analysis. Gender-specific differences were analysed by calculating the $95 \%$ confidence intervals for women and men separately. These were subtracted (men - women) for significance testing. Finally, significant differences were normalized by dividing each feature by appropriate distance measurements and confidence intervals were recalculated. 


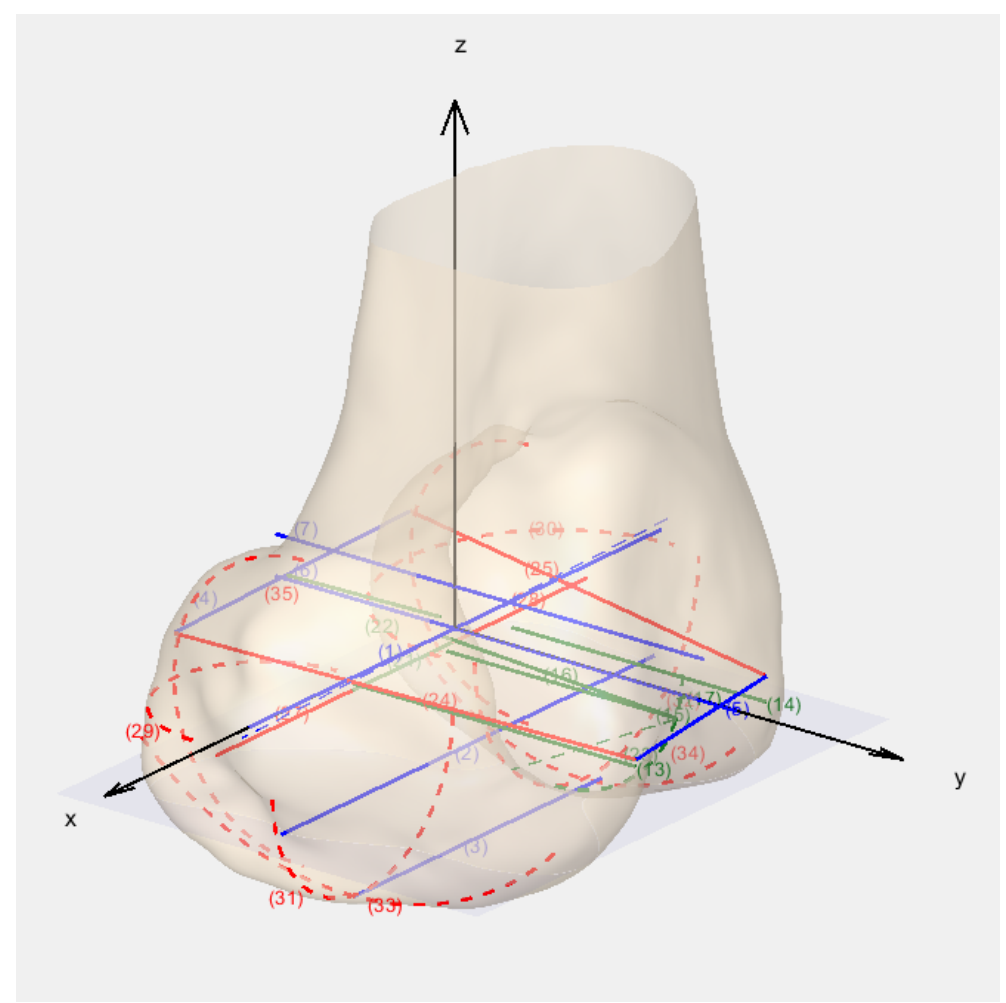

Figure 1: Exemplarily visualization of the distal femur including 34 features to quantify the morphology

\section{Results}

The analysis of the distribution showed that, with exception of the Medial Distal Frontal Radius, all features were normally distributed. The appropriate initial confidence intervals are presented in Figure 2 a). Here, 6 of 34 features, in particular, the Whiteside-Epicondylar Angle, Trochlear Orientation in Frontal Plane, Sulcus Angle, Medial/Lateral Inclination and Lateral Distal Sagittal Radius did not show any significant differences between genders. Remarkably, this primarily involves angular (relative) features whereas distance (absolute) measurements were mostly gender dependent.

Regarding the normalization of significant different features, initially, each feature was divided by the Epicondylar Width as proposed by Mensch and Amstutz (Mensch and Amstutz, 1975). However, the recalculation of the confidence intervals showed that some differences could not be eliminated, especially those measured in AP direction. Therefore, we normalized all distance measurements and radii according to their direction of measurement: Features defined in ML direction were divided by the Epicondylar Width and those following the AP direction were normalized based on the Overall Anteroposterior Length. The results are presented in Figure $2 \mathrm{~b}$ ) and demonstrate that gender-specific differences mostly disappear by using an adequate normalization term. 
a)

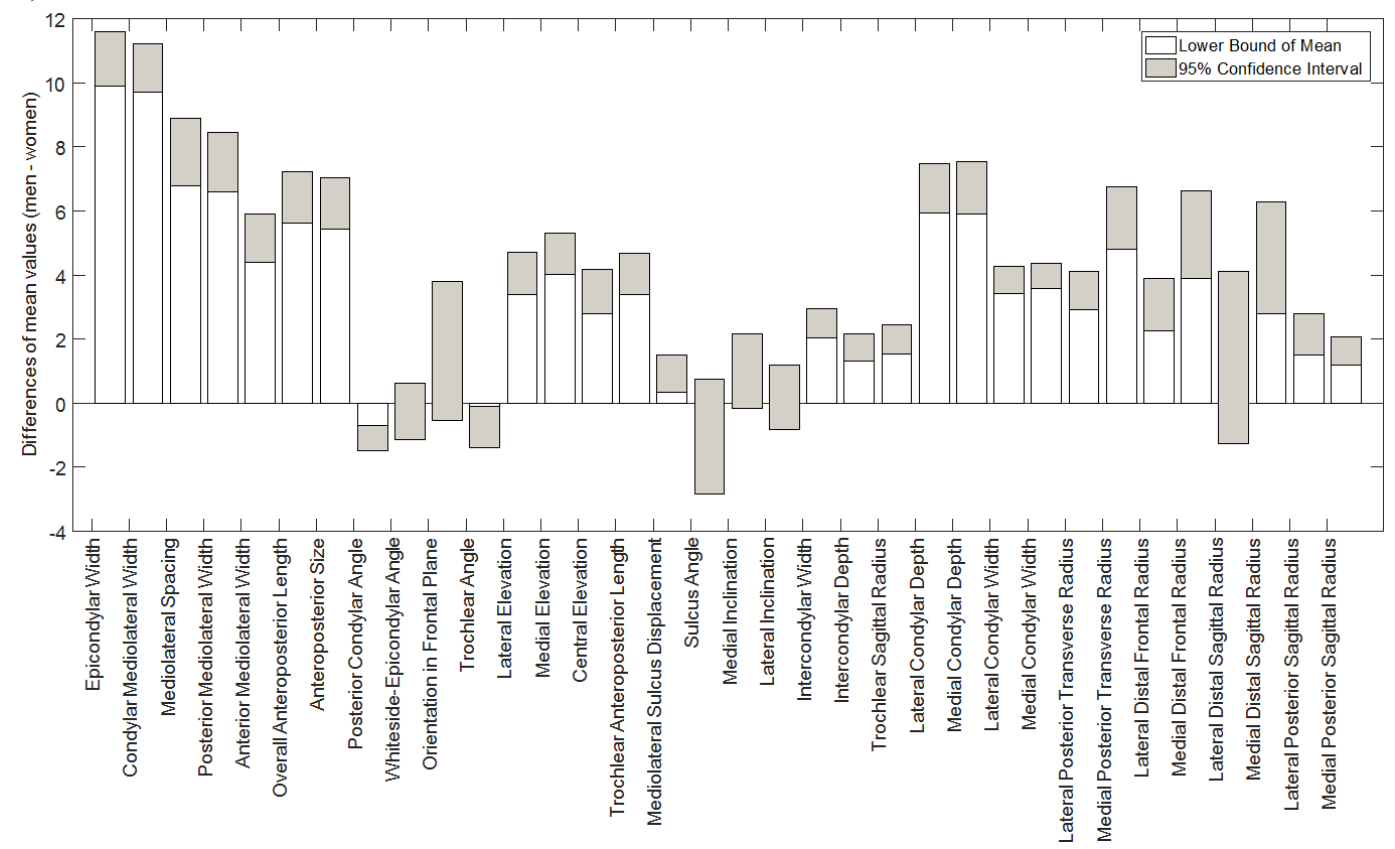

b)

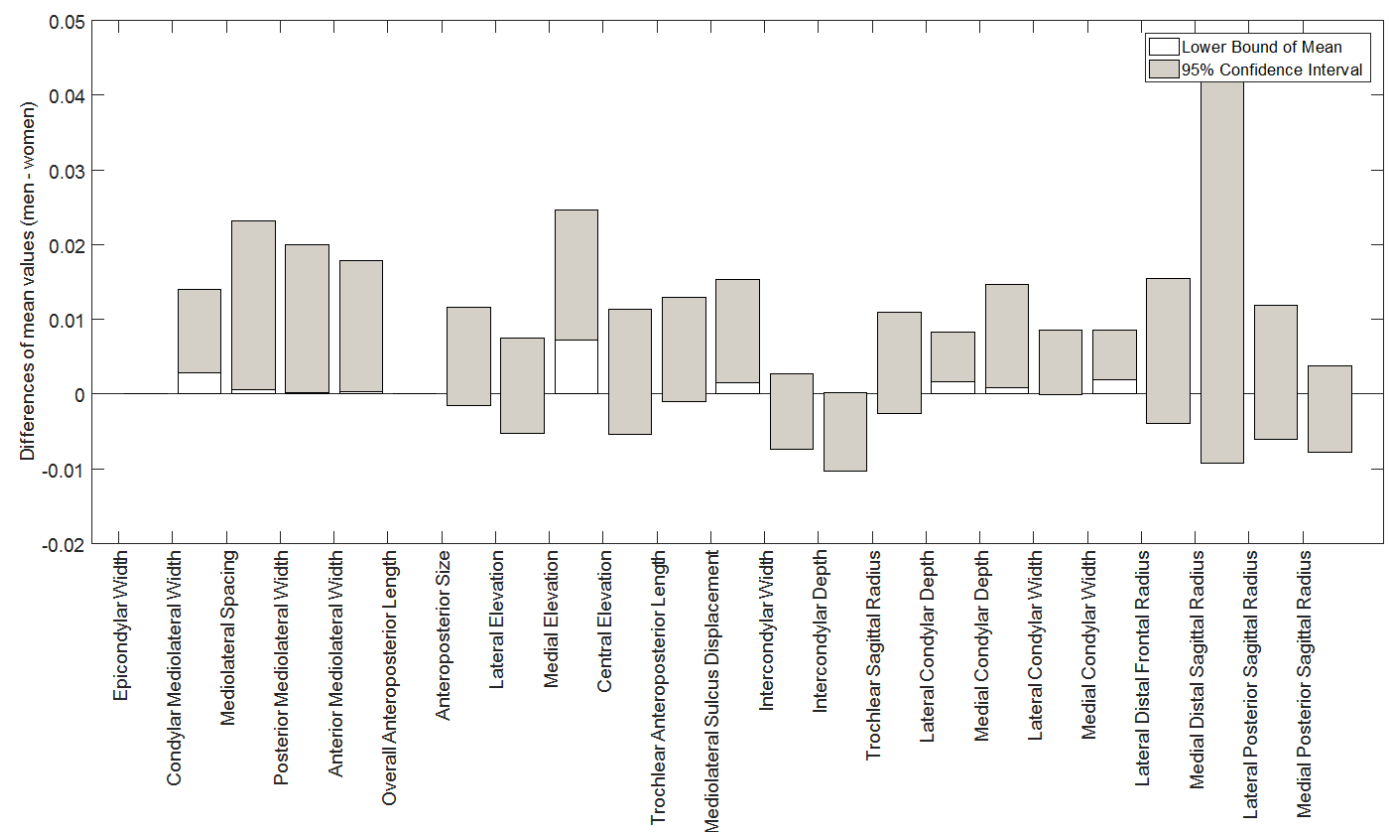

Figure 2: a) $95 \%$ confidence intervals (men - women) demonstrating gender-specific significant differences, b) $95 \%$ confidence intervals (men - women) after adequate normalization 


\section{Discussion}

Detailed knowledge on the knee morphology is essential for an optimal implant design representing one key factor for a successful therapeutic outcome. The statistical analysis of 34 features showed gender-specific differences, particularly in all distance measurements. In contrast, angular measurements did not show any or little gender dependency. Women had overall smaller femora and narrower dimensions at the same depth compared to men, which coincides with literature (Bellemans et al., 2005; Gillespie et al., 2011; Guy et al., 2012; Yang et al., 2014). In conclusion, a single feature for normalization seems to be insufficient to take gender differences in morphology into account. However, when considering ML and AP directed features separately, gender-specific differences disappear or were very small (much smaller than confidence intervals).

In conclusion, implant sizes (femoral components) should not be linearly scaled according to one dimension as proposed e.g. by Mensch and Amstutz (Mensch and Amstutz, 1975). Instead, ML and AP directions should be regarded separately (non-isotropic scaling). Taking this into consideration, gender-specific differences might be neglected. Finally, high standard deviations and confidence intervals demonstrated that the distal femoral morphology is highly individual and therefore should be treated rather patient-specific than gender-specific. Further investigations of the relationship between the implant design, patient-specific biomechanical situation and the associated clinical outcome is part of our ongoing work.

\section{References}

Asseln, M., Hanisch, C., Al Hares, G., Eschweiler, J., Radermacher, K., 2016. Automatic parameterisation of the distal femur based on 3D surface data: a novel approach for systematic morphological analysis und optimisation. Bone \&amp; Joint Journal Orthopaedic Proceedings Supplement 98-B, 31.

Bellemans, J., Ries, M.D., Victor, J.M. (Eds.), 2005. Total Knee Arthroplasty. A Guide to Get Better Performance. Springer Medizin Verlag Heidelberg, Berlin, Heidelberg.

Gillespie, R.J., Levine, A., Fitzgerald, S.J., Kolaczko, J., DeMaio, M., Marcus, R.E., Cooperman, D.R., 2011. Gender differences in the anatomy of the distal femur. The Journal of bone and joint surgery. British volume 93, 357-363.

Guy, S.P., Farndon, M.A., Sidhom, S., Al-Lami, M., Bennett, C., London, N.J., 2012. Gender differences in distal femoral morphology and the role of gender specific implants in total knee replacement: a prospective clinical study. The Knee 19, 28-31.

Hitt, K., Shurman, J.R., Greene, K., McCarthy, J., Moskal, J., Hoeman, T., Mont, M.A., 2003. Anthropometric Measurements of the Human Knee: Correlation to the Sizing of Current Knee Arthroplasty Systems. J Bone Joint Surg Am 85, 115.

Mensch, J.S., Amstutz, H.C., 1975. Knee morphology as a guide to knee replacement. Clinical orthopaedics and related research, 231-241.

Parsons, F.G., 1914. The Characters of the English Thigh-Bone. Journal of Anatomy and Physiology 48, 238-267.

Yang, B., Yu, J.-K., Zheng, Z.-Z., Lu, Z.-H., Zhang, J.-Y., 2014. Comparative study of sex differences in distal femur morphology in osteoarthritic knees in a Chinese population. PloS one 9, e89394. 


\section{Disclosures}

This work has been supported in parts by Conformis, Inc., Bedford, USA. 